from women. Serology was offered for HIV and Syphilis. Hepatitis B serology was offered to those who reported intravenous drug use or sex with commercial sex workers. Anyone diagnosed with an STI was offered treatment at Occupational Health services on site or a local sexual health clinic.

Results In total 614 clients were seen, with a median age of 28 years. $91 \%$ were men. There were 19 different ethnic groups. Nearly half (285/614, 46\%) reported their ethnic group as English/Scottish/ Welsh. The other large ethnic groups were 53 Indian (9\%), 52 Eastern European (8\%) and 39 Irish (6\%). 20 clients (3\%) had Chlamydia and one was diagnosed with Hepatitis B. Reported levels of sexual risk factors are shown in abstract P101 table 1.

Abstract P101 Table 1 Sexual risk factors of in the olympic site construction workers

\begin{tabular}{ll}
\hline Sexual risk factor & No. of people/ \% of sample \\
\hline Sex between men & $5(<1 \%)$ \\
Previous STI & $20(3 \%)$ \\
More than one sexual partner in last 3 months & $173(28 \%)$ \\
Intravenous drug use & $3(<1 \%)$ \\
Use of commercial sex workers & $3(\mathrm{n}=76)(4 \%)$ \\
\hline
\end{tabular}

Conclusions There was a low prevalence of STIs in these construction workers, contradicting prior fears of a high disease burden. Reported levels of sexual risk factors, including use of commercial sex workers, were low.

\section{P102 A HIGHLY COST-EFFECTIVE AND TARGETED SERVICE PROMOTION CAMPAIGN USING THE SOCIAL MEDIA SITE FACEBOOK}

doi:10.1136/sextrans-2012-050601c.102

\section{S Day, A Hughes.* Chelsea and Westminster Hospital, London, UK}

Background Facebook is the leading social media site in the world with over 30 million active users in the UK, $50 \%$ of who log on every day. It enables promotion to be targeted to specified demographic groups and is cost-effective because advertisers only pay for users who click through to their website. Using Facebook we led an advertising campaign to men having sex with men (MSM).

Method The advert featured from 9th October to 31st December 2009 and offered a free rapid HIV testing and sexual health screening service to users actively logged on to their Facebook account. We targeted men interested in men, aged between 18 and $50 \mathrm{yrs}$, that were single or in a relationship and living within 50 miles of our city. Users clicking onto the advert were automatically diverted to our website's appointments section. Each click-through incurred a small fee ("Pay-per-click").

Results The campaign targeted 30580 registered Facebook MSM users. Over 3 months the advert appeared 2.86 million times. Up to 9750 individuals were exposed to the advert on any given day and it frequently featured several times a day to each user. In total, 872 $(2.9 \%)$ registered MSM users clicked through to our website at an average cost of $£ 0.55$ per click. These were aged 18-24 (55\%), 25-34 (27\%), 35-44 (14\%) and 45-54 (4\%) years. The total cost of the campaign was $£ 483$. Facebook provided regular statistical reports which enabled us to monitor its effect, control our budget and prospectively tailor the advert accordingly.

Discussion This simple Pay-per-click Facebook campaign advertised to $8.5 \%$ of the UK MSM population (estimated UK MSM population is 3.6 million). Using Payment by Results income, only $0.3 \%$ (3-4) of clickers would need to attend our service to make the campaign costneutral. Whilst the functionality, diversity and social reach of Face- book is growing year on year, this presents a highly cost-effective opportunity for service/health promotion to hard to reach groups.

\section{Electronic patient records and use of IT P103 ENHANCING PATIENT SAFETY IN A LARGE HIV OUT- PATIENT SERVICE: EVALUATION OF AN ELECTRONIC RESULTS CHECKING SYSTEM FOR BLOOD TESTS}

doi:10.1136/sextrans-2012-050601c.103

D Nugent, ${ }^{*}$ N Uthayakumar, R Ferrand, S Edwards, P Benn. Mortimer Market Centre, London, UK

Background HIV services face the challenge of regular monitoring of a growing patient cohort and ensuring prompt action upon abnormal results. We identified a number of issues using a paperbased results system (PBS) including: (1) missing results, (2) delayed delivery, (3) clinician error, (4) lack of audit trail, placing patients at risk of delayed identification of drug toxicity and serious conditions for example, acute hepatitis.

Aims We piloted an electronic results checking system (ERC) which classifies results as normal or abnormal (non-urgent (NUAbn) or urgent (UAbn)) to compare the speed and performance of PBS and ERC in identifying biochemical abnormalities.

Methods Between 4 July 2011 and 22 July 2011 we compared the time intervals from sampling to (1) receipt of results; (2) clinician review of UAbn/NUAbn and (3) review of NUAbn by a clinician. Abnormalities were graded and both systems reviewed daily. Data were analysed using STATA V.11.0. Mann-Whitney U tests were used to compare the intervals.

Results Of 513 patients undergoing $\geq$ one blood test, $296(57.7 \%)$ had $\geq$ one biochemical abnormality identified by the ERC $(10.7 \%$ UAbn, $42.3 \%$ NUAbn and $47 \%$ not clinically significant). Of these, PBS simultaneously identified $83 \%$. The median interval between sampling to (1) receipt of results was 1 (IOR $1-2$ ) vs 4 days (IOR 3-5), $\mathrm{p}<0.0001$; (2) clinician review 3 (IOR 1-4) vs 3 (IOR 3-6) days, $\mathrm{p}<0.037$; and (3) review of NUAbn by clinician 2 (IOR $1-4$ ) vs 10 days (IOR 9-12), $\mathrm{P}=0.136$, for ERC and $\mathrm{PBS}$ respectively. $11 \%$ of the missing PBS results were classified UAbn. ERC missed three abnormalities highlighting a software error which has now been corrected.

Conclusion We demonstrate the use of IT to review blood results leads to the faster identification of biochemical abnormalities, which are common in our HIV cohort, facilitating their timely management. We anticipate the use of ERC in routine practice will avoid delay/non-identification of a significant number of abnormal results within our service.

\section{P104 PATIENT VIEWS ON CONFIDENTIALITY IN THE ELECTRONIC ERA}

doi:10.1136/sextrans-2012-050601c.104

E Stachow, ${ }^{*}$ A Wong, Z Soogun-Shah, S Barrett. Birmingham Heartlands Hospital

Background Confidentiality is a cornerstone of good medical practice. However, sharing of medical information is often essential for safe, effective care. The advent of computerised records has increased the scope for efficient data transfer. However, the same principles for patient confidentiality remain for this format. Ambiguity in current guidelines exists regarding patient data relating to sexual health. Peer-reviewed research reveals a lack of patient understanding on confidentiality, and concerns on inappropriate data disclosure within local areas. 